Raise a glass

Nature Commun. 5, 4219 (2014)

Techniques using inelastic photon or neutron scattering provide access to atomic and molecular motion or collective excitations across a range of time and length scales. When it comes to understanding glass transitions, however, their impact is limited as no probe currently provides access to the appropriate region of time-length space.

But now Yuri Shvyd'ko and colleagues have devised a method to explore this uncharted territory. By combining flat crystal angular dispersive optics with focusing and collimating optics, they have built an ultra-high-resolution inelastic X-ray scattering spectrometer that can bridge the nano- to mesoscale.

Shvyd'ko et al. demonstrated the potential of their technique with studies of the dynamics in glycerol, a prototypical glass-forming liquid. But their method - which should be suitable for most X-ray synchrotron and free-electron laser facilities - opens up a new region of the dynamics landscape, with implications beyond glass physics. And with a few additions, this approach could be developed to enable single-shot measurements, further enhancing spectroscopic performance.

\section{Back from the future \\ Nature Commun. 5, 4145 (2014)}

A closed time-like curve is a theoretically allowed spacetime trajectory that forms a loop - the end being the same point in space and time as the start. As if this doesn't seem weird enough, give quantum computers access to systems that are trapped in such a curve and things start to get even wilder.

The key point is that by intertwining the past and the future of a quantum system, closed time-like curves induce a nonlinear quantum evolution. And - as has now been experimentally simulated by Martin Ringbauer and colleagues - this non-linearity may be capable of overriding some of the most basic assumptions of quantum mechanics, including the ideal case of perfect discrimination of nonorthogonal states.

As table-top wormholes are not (yet) available, the experiment simulated a single photon locked in a closed time-like curve by ensuring that its initial and final states were exactly the same. A second photon, interacting with the first, was then shown to undergo the predicted nonlinear dynamics effectively constituting one of the first examples of how relativistic phenomena could influence quantum physics.

FL

\section{Spin for heat}

Phys. Rev. Lett. 113, 027601 (2014)

A temperature difference between two metals in contact generates a voltage - the Seebeck effect. The opposite phenomenon, known as the Peltier effect, implies heating or cooling at the interface of such a junction when an electric current flows through it. The two processes are Onsager reciprocals, as they obey the reciprocal relations derived by Lars Onsager for irreversible thermodynamic processes.

Now, similar thermoelectric effects for spin currents are being explored. Following the discovery of the spin Seebeck effect at the interface between a magnetic insulator and a metal, Joost Flipse and colleagues now report its Onsager reciprocal, the spin Peltier effect.

The authors' device consists of a platinum film grown on top of $\mathrm{Y}_{3} \mathrm{Fe}_{5} \mathrm{O}_{12}$ (yttrium iron garnet, an insulating ferrimagnet). A charge current through the platinum layer, in combination with an external magnetic field, results in spin accumulation at the interface - the spin Hall effect. This spin build-up is picked up by the magnetic insulator and converted into magnons (collective excitations of

\title{
Local hot bubble
} Nature http://dx.doi.org/10.1038/nature13525 (2014)

The origin of a million-degree bubble of ionized gas surrounding the Sun is hotly debated. Is the gas produced within the Solar System, or is it of interstellar origin? Such details are important for models of star and galaxy formation, for example. Massimiliano Galeazzi and colleagues have used data from the Diffuse X-ray Emission from the Local Galaxy (DXL) space mission to quantify how much $X$-ray emission is locally sourced.

A volume of neutral hydrogen (and about 15\% helium) gas within a small interstellar cloud intersecting the Solar System can become ionized through the interaction with the charged solar wind, in a process known as charge exchange. When an electron returns to the ground state of an ion, soft X-rays are emitted. This type of gas forms a cone shape downstream from the Sun. Interstellar gas is not similarly focussed.

Analysis of the DXL data shows that roughly $40 \%$ of the soft X-ray flux is produced by charge exchange. This means that most of the flux is from hot interstellar plasmas rather than from the solar wind.

the crystal lattice's spin structure). Heat is transferred from the electrons in the platinum film to the magnons - or vice versa, depending on the direction of the magnetization in $\mathrm{Y}_{3} \mathrm{Fe}_{5} \mathrm{O}_{12}$.

\section{Protein power}

Proc. Natl Acad. Sci. USA http://doi.org/trv (2014)

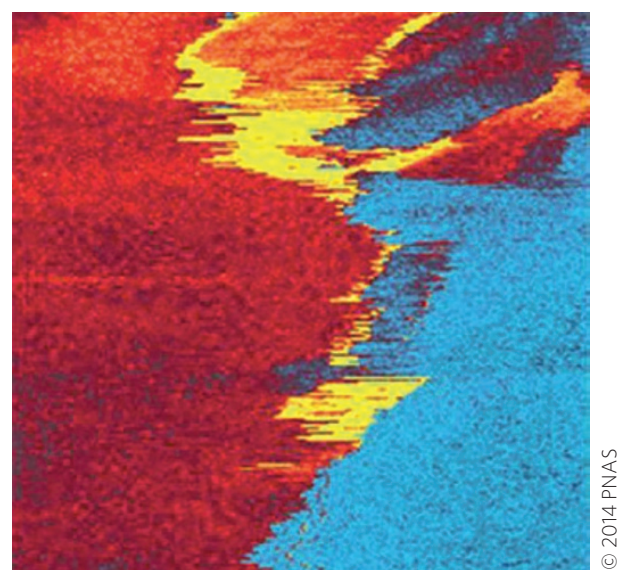

The protein elastin is best known for its mechanical properties: it's the reason our cheeks spring back into shape quickly after being pinched. But what of its electrical response? Yuanming Liu and colleagues have found macroscopic evidence of ferroelectric switching in elastin, and hit upon a model that recovers the polarization reversal underpinning this behaviour.

The same group previously reported an observation of ferroelectric switching in elastin and showed that it could be suppressed by glucose, but so far neither a macroscopic measurement nor a microscopic understanding of the underlying physics has been forthcoming. In the present study, Liu et al. used a combination of piezoresponse force microscopy measurements (pictured), molecular dynamics simulations and coarse-grained statistical mechanics to show that elastin retains its ferroelectric switching behaviour at high temperatures - its Curie temperature exceeding that of synthetic molecular ferroelectrics. This allowed them to probe the polarization of elastin on a macroscopic scale using pyroelectric current measurements.

The results of their simulation suggest that elastin polarization is strong even at the monomer level, much like the unit-cell polarization in perovskite ferroelectrics. And a structural model of elastin implicates the collective effect of aligned dipolar monomers in inducing the observed polarization.

$A K$

Written by May Chiao, Luke Fleet, Abigail Klopper, Federico Levi and Bart Verberck 